\title{
A metric-based assessment of flood risk and vulnerability of rural communities in the Lower Shire Valley, Malawi
}

\author{
A. J. Adeloye ${ }^{1}$, F. D. Mwale $^{2}$, and Z. Dulanya ${ }^{3}$ \\ ${ }^{1}$ Institute for Infrastructure and Environment, Heriot Watt University, Riccarton, Edinburgh, EH14 4AS, UK \\ ${ }^{2}$ The Polytechnic, Dept. of Civil Engineering, University of Malawi, PMB 303, Blantyre 3, Malawi \\ ${ }^{3}$ Dept. of Geography and Earth Sciences, University of Malawi, P.O. Box 280, Zomba, Malawi \\ Correspondence to: A. J. Adeloye (a.j.adeloye@hw.ac.uk)
}

Received: 11 March 2015 - Accepted: 11 March 2015 - Published: 11 June 2015

\begin{abstract}
In response to the increasing frequency and economic damages of natural disasters globally, disaster risk management has evolved to incorporate risk assessments that are multi-dimensional, integrated and metricbased. This is to support knowledge-based decision making and hence sustainable risk reduction. In Malawi and most of Sub-Saharan Africa (SSA), however, flood risk studies remain focussed on understanding causation, impacts, perceptions and coping and adaptation measures. Using the IPCC Framework, this study has quantified and profiled risk to flooding of rural, subsistent communities in the Lower Shire Valley, Malawi. Flood risk was obtained by integrating hazard and vulnerability. Flood hazard was characterised in terms of flood depth and inundation area obtained through hydraulic modelling in the valley with Lisflood-FP, while the vulnerability was indexed through analysis of exposure, susceptibility and capacity that were linked to social, economic, environmental and physical perspectives. Data on these were collected through structured interviews of the communities. The implementation of the entire analysis within GIS enabled the visualisation of spatial variability in flood risk in the valley. The results show predominantly medium levels in hazardousness, vulnerability and risk. The vulnerability is dominated by a high to very high susceptibility. Economic and physical capacities tend to be predominantly low but social capacity is significantly high, resulting in overall medium levels of capacity-induced vulnerability. Exposure manifests as medium. The vulnerability and risk showed marginal spatial variability. The paper concludes with recommendations on how these outcomes could inform policy interventions in the Valley.
\end{abstract}

\section{Introduction}

With increasing frequency, severity and associated damages of natural disasters, disaster management has also evolved to underscore multi-dimensioning, integration and the use of quantitative metrics for risks (Birkmann, 2006, 2007). Metric-based assessments of risk make possible to compare the relative risks of specific people and places, and thus the prioritisation of allocation of limited financial and other resources. They also enable the monitoring of the progress of policy interventions (Gall, 2007). This is pertinent to resource-strapped communities in SSA where droughts and floods alone account for 80 and $70 \%$ of disaster related mortality and economic losses respectively (World Bank, 2010a).
The current study contributes to the understanding of flood risk and its quantification for rural communities in SSA by analysing the Lower Shire Valley, Malawi as a case study. It adopts a radical departure from previous attempts that have focused on one of either the social vulnerability or physical hazard but not the interplay between these two aspects.

In the next Section, the case study and adopted methodology are described. Next comes the results and discussions, followed finally by the main conclusions. 


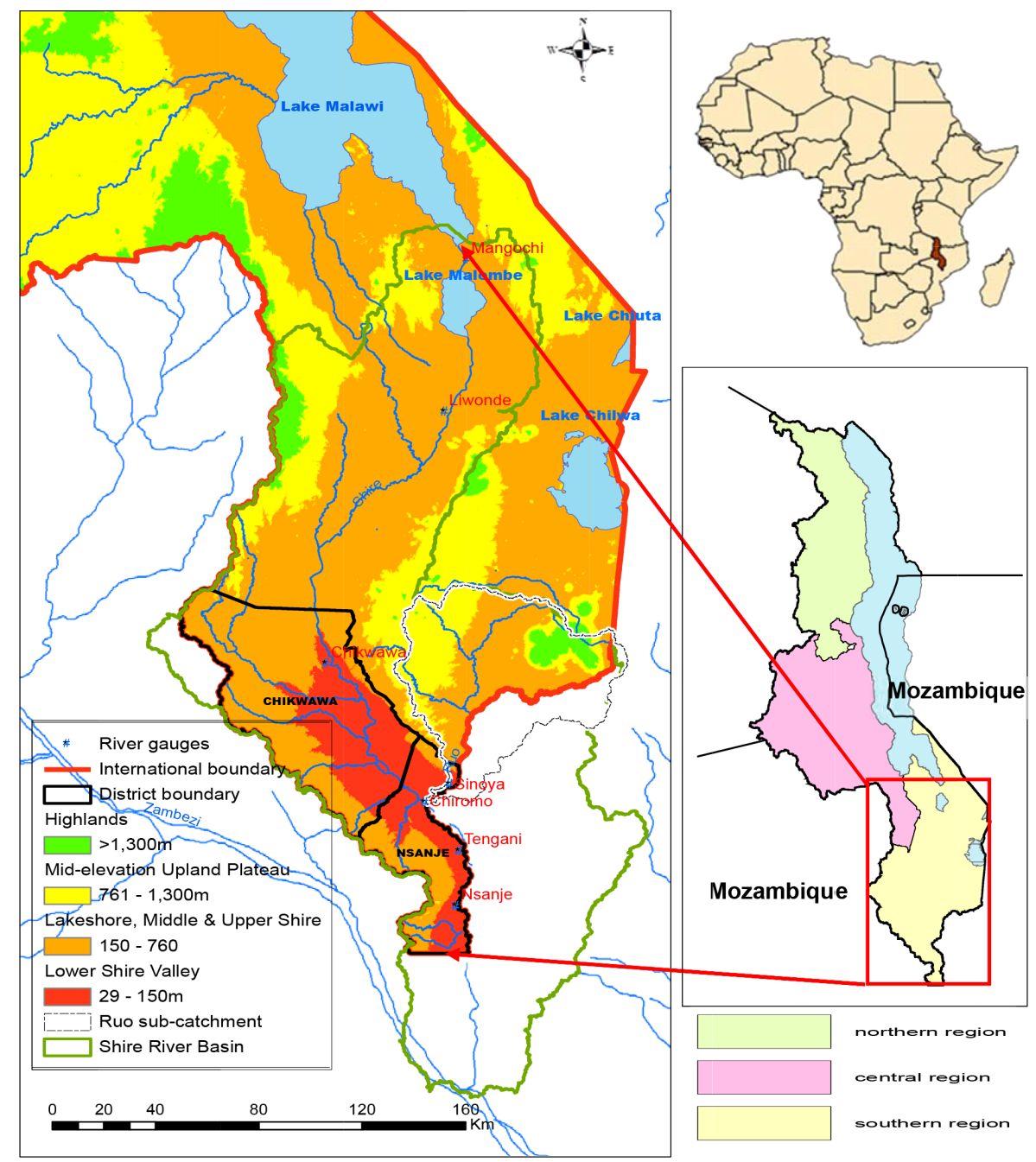

Figure 1. Geographical location of the study area.

\section{Case study area and methods}

\subsection{The study area}

The Lower Shire Valley lies at the southern tip of Malawi and straddles two administrative districts - Chikwawa and Nsanje - and is part of the Shire Basin, which extends into Mozambique (Fig. 1). Annual rainfall in the basin is highly spatially variable, reaching about $2000 \mathrm{~mm}$ in the eastern part due to orographic influences. This often results in high flows of up to $5400 \mathrm{~m}^{3} \mathrm{~s}^{-1}$ at the Sinoya station on the Ruo River, causing devastating floods particularly in areas near its confluence with the main Shire River (Shela et al., 2008).

Poverty rates in the two districts are the highest in the country, with over $80 \%$ living on less than USD 0.4/capita/day (National Statistical Office, 2012). Livelihoods are derived from subsistence farming, livestock rearing and casual labour, all of which are threatened by a multiplicity of stressors including floods, droughts and HIV/AIDS, among others (Casale et al., 2008).

\subsection{Some basic definitions and scale of analyses}

Risk is associated with potential loss arising from the interactions of vulnerable conditions and the hazard (ISDR, 2009). Hazard is a dangerous phenomenon or activity that may cause loss of tangible (e.g. life) and intangible (e.g. services) things. Vulnerability is the intrinsic disposition of a system to harm, often determined by its exposure, susceptibility and capacity/resilience (Birkmann et al., 2013).

Vulnerability, hazard and consequently risk, have temporal and spatial scale ramifications; indeed the choice of an appropriate spatial scale significantly affects the effectiveness of disaster risk reduction interventions. In particular for the poor communities of the Shire basin, better information can be obtained by operating at the local scale because the impact of the flooding is mostly felt at this scale. In this regard for 
Malawi, the relevant local scale to use thus requires careful considerations.

While the Administrative scales (Chikwawa and Nsanje) might look appropriate, these were considered too coarse to capture the risks faced by local communities. On the other hand, the village development committees into which the villages are organised were considered too fine and the cost of collecting the data at such a fine scale might be prohibitive. As a compromise, the Area Development Committee (ADC) levels were selected as the scale for the study. Chikwawa has 11 ADCs while Nsanje has 9; each ADC is headed by a Chief, the Traditional Authority (TA). In general, developmental work in Malawi through NGOs and others is also much tied to administrative boundaries of TAs (Malcomb et al., 2014).

\subsection{Theoretical development of indices}

\section{Vulnerability}

To measure vulnerability, following an extensive review of indices for developing countries, the study adapted the Community Based Disaster Risk Index (CBDRI). In its original form, the CBDRI is (Bollin et al., 2003):

$$
\mathrm{CBDRI}=\lambda(H+E+S-C)
$$

$$
H=\sum_{i=1}^{h} w_{i} x_{i} ; \quad E=\sum_{j=1}^{q} w_{j} x_{j} ;
$$

$S=\sum_{k=1}^{r} w_{k} x_{k} ; C=\sum_{y=1}^{z} w_{y} x_{y}$

where $H, E, S$ and $C$ are the hazard, exposure, susceptibility and capacities (resilience) sub-components (each ranging from 0 and 100); $h, q, r, z$ are the total number of variables in the hazard, exposure, susceptibility and capacity components respectively; $x$ is a score allocated to the variable in the sub-component based on the answer from the community and is equal to either 1 (low), 2 (medium) or 3 (high); $w$ is the weight attached to each score based on respondents perception of the importance of a given variable (the $w$ 's summed over all the factors for each sub-component $=33$ ); and $\lambda(=0.33)$ is a factor that helps ensure that the final index also lies in the interval $[0,100]$.

As the name implies, CBDRI was developed to measure risk directly; however, it is being adapted here for indexing vulnerability which has been made possible by its additive form as shown in Eq. (1). The adaptation of the index carried out in the current study was to quantify the vulnerability from the $E, S$ and $C$ components and aggregate these to the total vulnerability using the arithmetic aggregation scheme as follows (Allison et al., 2009; Hahn et al., 2009):
$V=\frac{1}{3}[E+S+(1-C)]$

where $(1-C)$ represents the lack of capacity. Once obtained, the vulnerability (expressed in decimal fraction) was then categorised as follows: $0-0.2=$ very low, 0.21 $0.4=$ low, $0.41-0.6=$ medium, $0.61-0.8=$ high and $0.81-$ $1.0=$ very high

Table 1 contains a partial list of the factors considered for the CBDRI evaluation; the complete list is provided by Mwale (2014).

\subsection{Measuring the hazard}

The hazard was characterised in terms of the depth (m) of flood inundation as determined from the hydraulic modelling of the valley using Listflood-FP (Bates and De Roo, 2000). The model was applied to the $2007 / 2008$ flood season because the associated daily flow data at Chiromo on the Shire (see Fig. 1) were the most recent at the time of the study. The $90 \mathrm{~m}$ STRM DEM was used; this was re-sampled to $270 \mathrm{~m}$ to reduce computation time. Manning's $\mathrm{n}$ values tested fell in the range of 0.025 to 0.07 as recommended by Chow (1959) for the dominant land cover in the valley that comprises savannah, herbaceous and degraded agricultural fields (Fernandes et al., 2006). The value of the $\mathrm{n}$ adopted for the final model was based on assessment of the performance of the model using the similarity index (Bates and De Roo, 2000).

The severity of flood hazard (m) was based on the designation by Dinh et al. (2012): $0-0.2=$ very low, 0.2 $0.5=$ low, $0.5-1.0=$ medium, $1.0-2.0=$ high $;>2.0=$ very high. Where, as expected, a community has many hazard zones (or severities), these were combined to obtain an average hazard index (AHI) using:

$\mathrm{AHI}=\frac{\sum_{i=1}^{n}(\mathrm{HI})_{i} A_{i}}{\sum_{i=1}^{n} A_{i}}$

where $A_{i}$ is the land area in flood hazard class $\mathrm{HI}_{i}(\mathrm{~m})$ and $n$ is the total number of hazard zones in the community.

\subsection{Measuring flood risk}

The flood risk $(R)$ was calculated as a convolution of the hazard $(H)$ and vulnerability $(V)$ through:

$R=H \times V$

after the hazard class has been made dimensionless by scaling the depths in the hazard class by $3.3 \mathrm{~m}$, representing the maximum depth simulated by LisFlood. The resulting risk classes are: $0-0.012$ (very low); 0.012-0.06 (low); 0.06-0.18 (medium); 0.18-0.50 (high); and $>0.50$ (very high). 
Table 1. Vulnerability factors in the CBDRI.

\begin{tabular}{|c|c|c|}
\hline Component & Indicator name & Indicator \\
\hline \multicolumn{3}{|c|}{ Exposure } \\
\hline Structure & $\begin{array}{l}\text { (E1) No. of housing units } \\
\text { (E2) Lifelines }\end{array}$ & $\begin{array}{l}\text { No. of housing units (living quarters) } \\
\% \text { of homes with piped drinking water }\end{array}$ \\
\hline Population & (E3) Total resident population & Total resident population \\
\hline Economy & (E4) Local gross domestic product (GDP) & Total locally generated GDP in constant currency \\
\hline \multicolumn{3}{|c|}{ Susceptibility } \\
\hline Environmental & $\begin{array}{l}\text { (V14) Area under forest } \\
\text { (V15) Degraded land } \\
\text { (V16) Overused land }\end{array}$ & $\begin{array}{l}\% \text { area of the commune covered with forest } \\
\% \text { area that is degraded/eroded/desertified } \\
\% \text { of agricultural land that is overused }\end{array}$ \\
\hline \multicolumn{3}{|c|}{ Capacity and measures } \\
\hline Economic capacity & $\begin{array}{l}\text { (C11) Local emergency funds } \\
\text { (C12) Access to local emergency funds } \\
\text { (C13) Access to international emergency funds } \\
\text { (C14) Insurance market } \\
\text { (C15) Mitigation loans } \\
\text { (C16) Reconstruction loans } \\
\text { (C17) Public works }\end{array}$ & $\begin{array}{l}\text { Local emergency funds as } \% \text { of local budget } \\
\text { Release period of national emergency funds } \\
\text { Access to international emergency funds } \\
\text { Availability of insurance for buildings } \\
\text { Availability of loans for disaster risk reduction measures } \\
\text { Availability of construction credits } \\
\text { Magnitude of local public works programme }\end{array}$ \\
\hline
\end{tabular}

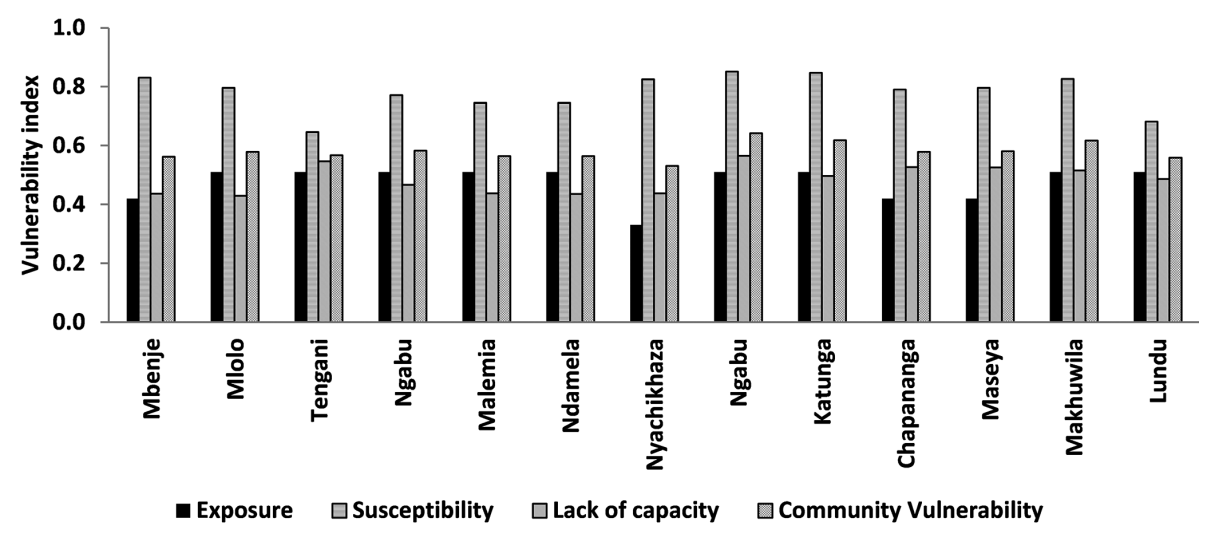

Figure 2. Vulnerability magnitudes from exposure, susceptibility and a lack of capacity across communities.

\section{Results and discussions}

\subsection{Vulnerability}

Data on components of the vulnerability were collected by administering questionnaires to 13 community groups (i.e. ADCs) in the Shire Valley. In total, 38 variables were included in the questionnaires; however, due to lack of space, these questionnaires and the scores/weights allocated by respondents are not being detailed here but are available on request (see also Mwale, 2014).

Figure 1 shows the resulting levels of vulnerability by exposure, susceptibility, capacities as well as aggregately, from where it is clear that aggregate community vulnerability is medium $(0.4-0.6)$ to high $(0.6-0.8)$ but predomi- nantly medium. Exposure tends to be to be medium; in contrast, susceptibility manifests as high to very high (0.8-1.0), thus dominating the vulnerability. The strong societal capacity within the communities has largely been responsible for tempering the capacity component of the vulnerability as shown in Fig. 2. Thus, although the communities lacked economic and physical capacity, most respondents scored the societal support, e.g. the existence of Civil Protection Committees, reasonable community participation, substantial awareness, use of sms, drumming and whistling for flood warning, etc. very highly. Such community strength has also been described by Nilson et al. (2010).

While there are differences in actual vulnerability scores between communities (see Fig. 2) there is a general pattern of homogeneity for a given dimension suggesting insubstan- 


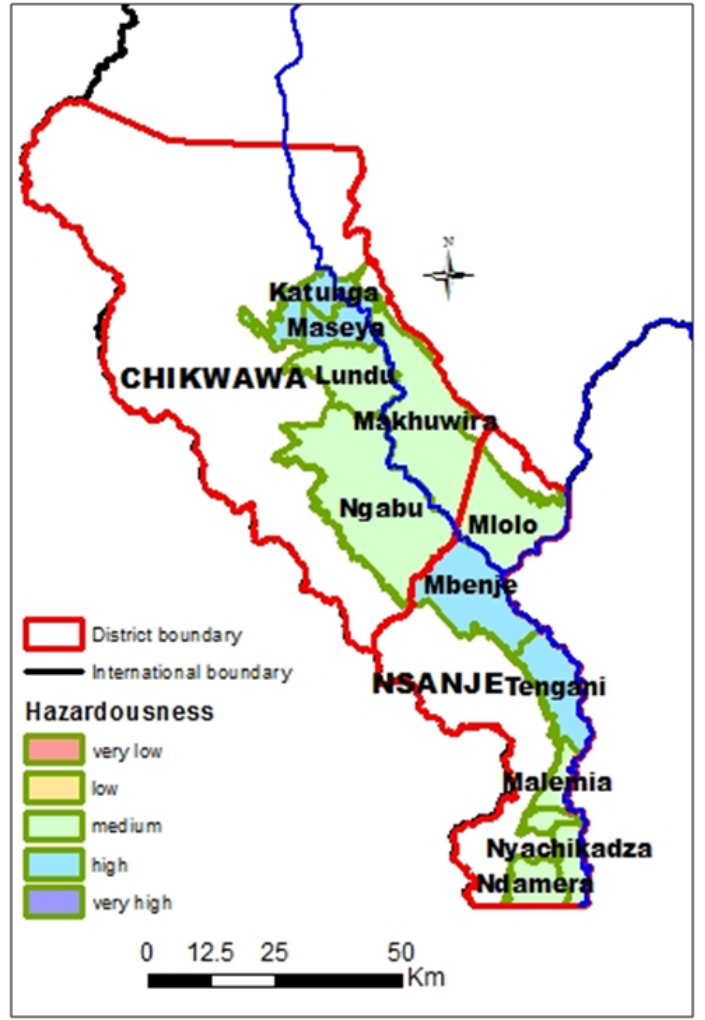

Figure 3. Spatial variation in flood hazardousness in the Lower Shire Valley.

tial differences between communities. This is not surprising given that both Nsanje and Chikwawa districts are very similar in their socio-economic profile and poverty levels, resulting in similar limited economic capacity for disaster management. Consequently, vast diversity in vulnerability in the Lower Shire valley at this scale is unexpected.

\subsection{Flood hazardousness}

Analysis of the similarity index for different Manning's $n$ (see Mwale (2014) for details) showed that the LisfloodFP model produced the most satisfactory fit with the MODIS remotely sensed image of the inundation extent of the 2007/2008 flood at $n=0.05$ (similarity index $=69 \%$ ). Lisflood-FP results for this $\mathrm{n}$ were thus used for characterising the hazard in the valley.

The average hazard index (AHI - estimated according to Eq. 4) has been plotted in Fig. 3 from where it is clear that the Lower Shire is a region affected by medium to high flood levels but with a predominance of the medium hazard class.

\subsection{Risk}

The overall risk profile is shown in Fig. 4 from where it is clear that communities in the Lower Shire Valley fall in the medium to high categories of the flood risk spectrum.

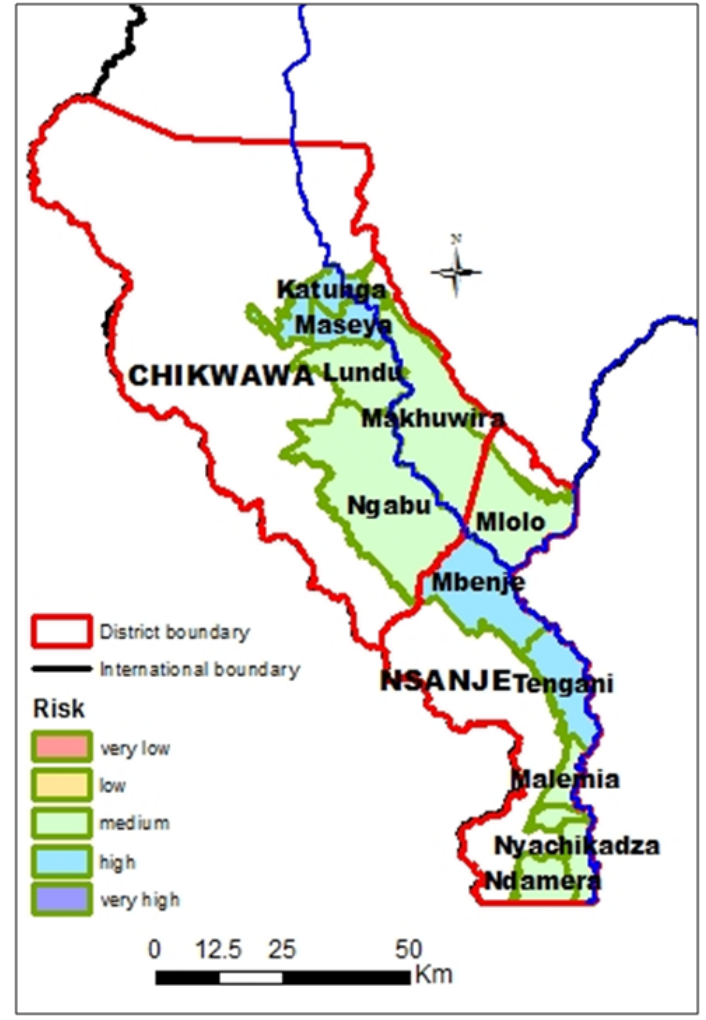

Figure 4. Spatial variation in risk in the Lower Shire Valley.

Medium risk communities are clearly dominant, accounting for 8 of the 12 communities assessed for risk, and arise from the medium levels of both the hazard and vulnerability. The few high risk communities, notably Katunga and Maseya in Chikwawa, and Mbenje and Tengani in Nsanje, are also those that suffer high hazardousness, a further reinforcement of the dominating influence of the hazard on the overall flood risk level in the valley.

\section{Conclusions}

This study has quantified and profiled vulnerability of rural communities in the Lower Shire Valley of Malawi as an example of the prevailing situation in much of SSA. It characterised vulnerability by exposure, susceptibility and capacity and then measured risk by integrating the vulnerability and hazard. While the vulnerability to flooding for rural communities in the Lower Shire Valley is medium to very high, there were significant variations in its three constituent dimensions. For example, although the susceptibility component was high to very high, the exposure component was medium. The lack of resilience or capacity is medium, which the study links to considerable societal capacity. Similarly, hazardousness and risk were predominantly medium for most communities in the valley; however, for the few areas where the hazard was high, the resulting risk was also high suggest- 
ing a strong influence of the hazard on the assessed risk. While there are differences in magnitudes across communities, these differences were marginal, an indication of relative homogeneity in flood risks in the communities. The vulnerability and risk magnitudes found in this study are thus consistent with magnitudes reported for rural communities in SSA, although measured in different ways and albeit to other climatological hazards, more specifically climate change (see e.g. Gbetibouo and Ringler, 2009; Malcomb et al., 2014).

There are, however, caveats that must be borne in mind. First is that the results are much dependent on variables in the index and their classification i.e. whether the variable is an exposure, susceptibility or capacity factor. Results are further affected by the thresholds set and the aggregation process applied. The spatial scale of application is another factor. Further, vulnerability data were sourced from communities which also make them potentially subjective. Likewise, hydrological and topographic data used in Lisfloof-FP hydraulic modelling are naturally subject to errors. The use of other vulnerability indices and risk assessment methods may therefore reveal different magnitudes and profiles. This is a problem nonetheless shared by all index-based vulnerability analyses. Further, the return period associated with the 2007/2008 event that formed the basis of the hazard evaluation was only 4-5 years (World Bank, 2010b); consequently, a radically intensified risk profile should be expected in the valley for rarer, high return period events, given the significant influence of the hazard on the overall flood risk as established in the current study.

Nonetheless, the outcome of this study is useful by potentially informing the formulation of more proactive policy interventions for redressing the incessant flooding and associated poverty intensification in the Lower Shire in particular and much of rural SSA in general. For example, the levels of susceptibility (high to very high) uncovered highlight the critical need for mainstreaming flood vulnerability reduction measures into the plethora of conventional developmental programmes taking place in SSA. This, to some extent, also addresses the issue of economic incapacity that arises from implementing lone flood risk reduction programmes. Given gross limitation in economic capacity and substantial societal capacity, programmes that will expand and strengthen societal capacity will provide the much needed leverage for risk reduction. Further, given the relative similar influence of hazardousness and vulnerability in the risk profile for most communities of the valley, there is the need for broad-based measures besides socio-economic adaptation. Finally, the apparent uniformity in risk levels across the Lower Shire communities suggests universal application of interventions to the floodplain.

Acknowledgements. The second author is grateful to the Malawi Government for the PhD Scholarship to undertake the reseearch study of which the work reported in this paper forms a part.

\section{References}

Allison, E. H., Perry, A. L., Badjeck, M. C., Adger, W. N., Brown, K., Conwal, D., Halls, A. S., Pilling, G. M., Reynolds, J. D., Andrew, N. L., and Dulvy, N. K.: Vulnerability of national economies to the impacts of climate change on fisheries, Fish Fisheries, 10, 173-196, 2009.

Bates, P. D. and De Roo, A. P. J.: A simple raster-based model for flood inundation simulation, J. Hydrol., 236, 54-77, 2000.

Birkmann, J. Measuring vulnerability to promote disaster-resilient societies:conceptual frameworks and definitions, in: Measuring vulnerability to natural hazards: Towards Disaster-Resilient Societies, edited by: Birkmann, J., United Nations University Press, Tokyo, New York, Paris, 9-54, 2006.

Birkmann, J.: Risk and vulnerability indicators at different scales: Applicability, usefulness and policy implications, Environ. Hazards, 7, 20-31, 2007.

Birkmann, J., Cardona, O. D., Carrenõ, M. L., Barbat, A. H., Pelling, M., Schneiderbauer, S., Kienberger, S., Keiler, M., Alexander, D., Zeil, P., and Welle, T.: Framing vulnerability, risk and societal responses: the MOVE framework, Nat. Hazards, 67, 193-211, 2013.

Bollin, C., Cardenas, C., Hahn, H., and Vasta, K. S.: Natural Disasters Network: Comprehensive Risk Management by Communities and Local Governments, Inter-American Development Bank, Washington, D.C., 2003.

Casale, M., Scott, D., Stuart, G., Suneetha, K., Paul, M., Tim, Q., and Gina, Z.: Experiencing vulnerability in southern Africa: The interaction of multiple stressors, Renewal Policy Brief, IFPRI, Washington, D.C., 2008.

Chow, V. T.: Open channel hydraulics, Mc-Graw Hill, New York, 1959.

Dinh, Q., Balica, S., Popescu, I., and Jonoski, A.: Climate Change Impact on Flood Hazard, Vulnerability and Risk of the Long Xuyen Quadrangle in the Mekong Delta, Int. J. River Basin Manage., 10, 103-120, 2012.

Fernandes, E. C. M., Gillison, A. N., Asner, G., and Richey, J.: Lower Zambezi River Basin: Baseline Data On Landuse, Biodiversity, And Hydrology, GEF - Zambezi Valley Market Led Smallholder Development Project, World Bank, Washington, D.C., 2006.

Gall, M.: Indices of social vulnerability to national hazards: a comparative evaluation, $\mathrm{PhD}$, University of South Carolina, Columbia, South Carolina, USA, 2007.

Gbetibouo, G. A. and Ringler, C.: Mapping South African Farming Sector Vulnerability to Climate Change and Variability - A Subnational Assessment, IFPRI Discussion Paper 00885, IFPRI, Wahington, D.C., USA, 2009.

Hahn, M. B., Riederer, A. M., and Foster, S. O.: The Livelihood Vulnerability Index: A pragmatic approach to assessing risks from climate variability and change - A case study in Mozambique, Global Environ. Change, 19, 74-88, 2009.

ISDR: Terminology on Disaster Risk Reduction, Geneva, Switzerland, 2009.

Malcomb, D. W., Weaver, E. A., and Krakowka, A. R.: Vulnerability modeling for sub-Saharan Africa: An operationalized approach in Malawi, Appl. Geogr., 48, 17-30, 2014. 
Mwale, F. D.: A contemporary management framework quantification of flood risk in rural Lower Shire Valley, Malawi, $\mathrm{PhD}$ thesis, Heriot Watt University, Edinburgh, UK, 2014.

National Statistical Office: Integrated Household Survey 2010 2011 - Household Socio-economic Characteristics Report, Zomba, Malawi, 2012.

Nilson, A., Shela, O. N., and Chavula, G.: Flood Risk Management Strategy: Mitigation, Preparedness, Response and Recovery, Department of Disaster Management Affairs, Malawi, 2010.
Shela, O. N., Thompson, G., Jere, P., and Annandale, G.: Analysis of Lower Shire Floods \& A Flood Risk Reduction and Recovery Programme Proposal for the Lower Shire Valley, Department of Disaster Management Affairs, Lilongwe, Malawi, 2008.

World Bank: Disaster Risk Reduction in Sub-Saharan Africa, Washington, D.C., 2010a.

World Bank: Malawi: Economic Vulnerability and Disaster Risk Assessment, Wahsington, D.C., USA, 2010b. 